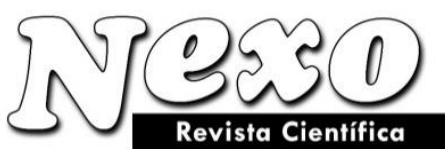

Vol. 34, No. 01, pp. 514-533/Marzo 2021

\title{
Improving the reliability of transport vehicles based on element-by-element analysis
}

\section{Mejoramiento de fiabilidad de medios de transporte por análisis de elemento por elemento}

\author{
Vladimir S. Volkov \\ Voronezh State University of Forestry and Technologies named after G. F. Morozov \\ *wl.volkov@yandex.ru
}

(recibido/received: 02-December-2020; aceptado/accepted: 20-February-2021)

\begin{abstract}
The author considers two approaches to solving the problem of increasing the reliability of machinebuilding products: on the spot, at the enterprises that operate transport vehicles, via certain modifications of serial products, and at manufacturing sites, during production of subsequent products, via improving the design on the basis of operation data of the analogues. In this case, data collection and processing system should provide reliable data on failures and cases of non-serviceable condition of the machines operating under different conditions; prompt processing of statistical data and presentation of results in the most convenient form; registration and coordination of reliability improvement measures taken by developers, manufacturers and operators. The author analyzes the operation of distribution laws of random variables and the variety of their corresponding calculation systems for the determination of the product's reliability indices in an element-by-element, unit-wise, as well as synthesized form. This approach makes it possible to search for the least reliable elements of a system in order to align the machine's reliability indices. Purpose. Creation of a design system for determining reliability indices in a separate form at the levels of parts, units and for the machine as a whole for identifying its least reliable components for the purpose of their further improvement. Methods. The methodology is based on drawing up a structural chart of machine reliability and a consolidated list of failures that are identified during its operation. The reliability structural scheme includes all the machine elements. A summary cumulative list is compiled further, where data on the operating time of each object are recorded for each failure type. The data samples on the times to failure of a group of homogeneous components are output from the summary cumulative list per each element. Results. The methodology makes it possible to create a system with multiple ratios of reliability indices per components, as well as reduce expenses for service impacts and improve the effectiveness of their transportation work. The present research work contributes to the theory of predicting the reliability of mechanical systems, making it possible to solve important tasks at modernization and design stages.
\end{abstract}

Keywords: Distribution law; Failure; Function; Information; Object; Reliability.

\section{RESUMEN}

Aquí se consideran dos enfoques básicos a mejorar la fiabilidad de productos de construcción mecánica, es decir, por unas mejoras de piezas seriales en las empresas que usan estos artículos y en el sitio de su 
fabricación durante la producción de muestras subsiguientes, así como a través de mejorar el diseño de las piezas sobre la base de los datos de funcionamiento de piezas similares anteriores. En este caso el sistema de recoger y procesar datos hará posible la adquisición de datos fiables sobre los fallos y casos de inoperabilidad de las máquinas usadas en condiciones de operación diferentes; procesamiento operacional de las estadísticas y presentación de los resultados en una forma conveniente para analizar. Se analizan de elemento por elemento y unidad por unidad el impacto de las leyes de distribución de variables aleatorias y la variedad de los sistemas de cálculo correspondientes en la determinación de los índices de fiabilidad del producto, así como en una forma sintetizada en la máquina en conjunto. Este enfoque permite buscar los elementos menos fiables en un sistema mecánico para tomar la decisión acerca de ajustar los índices de fiabilidad de los componentes respectivo de la máquina. Objetivo: crear un sistema de diseño para determinar índices de fiabilidad de forma separada en la escala de componentes, unidades y la máquina en conjunto para identificar sus componentes menos fiables con el objetivo de su mejoramiento ulterior. El sistema de métodos aquí usada se base en elaborar el diagrama estructural de fiabilidad de la máquina y una lista consolidada de fallos identificados en su operación. Después se compila un registro cumulativo donde se recuerdan los tiempos medios antes de fallar de cada objeto examinado para cada tipo de fallos. Se extraen del registro los datos para cada elemento sobre los tiempos antes de fallar de un grupo de elementos monotípicos del grupo de máquinas bajo control. La metodología desarrollada permite diseñar construcciones de próxima generación y usar los datos operacionales sobre máquinas de transporte durante este proceso para definir acciones determinadas para crear un sistema con razones proporcionales de índices de fiabilidad por componentes, así como emprender medidas específicas para reducir los gastos para impactos de servicio y mejorar la eficacia de su trabajo de transporte.

Palabras claves: Ley de distribución; Fallo; Función; Información; Objeto; Fiabilidad.

\section{INTRODUCTION}

At present, the consumer of engineering products is looking forward to the improvement of product reliability by manufacturer. In the practice of machine-building industry, there exist two basic approaches (Maslovsky, 1984) to solving this problem: on the spot, at the enterprises that operate transport vehicles, via certain modifications of serial products, and at manufacturing sites, during production of subsequent products, via improving the design on the basis of operation data of the previous analogues. But both in the former and in the latter case, the manufacturer must know the weak (unreliable) spots in the design of machines (equipment), which can be obtained as a result of data analysis on failures during operation (Volkov, 1999). Thus, the main purpose of information gathering is timely provision of credible data on the reliability of mechanical and automation equipment as well as their elements in operation.

The completeness of information depends on the accuracy of description of failures and their causes, indicating failure detection time; failure external manifestation; time for failure searching and troubleshooting; type of a failed component, part; its place in the general structure; time between failures, etc. (Barash, 2004).

Data collection and processing system (GOST 16468-79) should ensure the timely receipt of complete and reliable data on the reliability of machines operating in different conditions; prompt processing of statistical data and presentation of results in the form most convenient for analyzing the reliability of machines; recording and coordination of reliability improvement measures taken by developers, manufacturers and operators.

The results of data collection and processing on the machines reliability and automation facilities should ensure the solution of the following tasks (Methodical Guidelines. Reliability in technology, 1989):

- finding the causes of failures and malfunction;

- identification of parts, assembly units and components that limit the reliability of machines;

- determining and adjustment of the specified reliability factors of machines and their components; 
- justification of spare parts consumption list, structure of repair cycle and frequency of diagnostics, as well as maintenance and repair of machines;

- determining the influence of operating conditions and modes on the reliability of machines;

- determination of the economic effectiveness of measures regarding the improvement of the reliability of machines and their elements.

A critical problem in the study of mechanical system reliability is the so-called unit or element-by-element unevenness (Volkov, 1999), when separate components of such a system are characterized by a shorter time to failure, or significantly exceeding the mean time between system failures. Units, aggregates, or a machine as a whole can be considered as a system concept.

\section{MATERIALS AND METHODS}

Various sources may be applied for an overall assessment of machines reliability. The following sources (Fig. 1) were used by the author in the course of work (Volkov, 1999).

The occurrence of failures in mechanical systems is due to combined effect of a large number of different factors - dynamic loads, operating modes, high temperatures, mechanical characteristics of materials and parts, humidity, environmental dust, etc. Therefore, failures are of random nature, and failure itself is treated as a random event (Amalitsky et al, 2002).

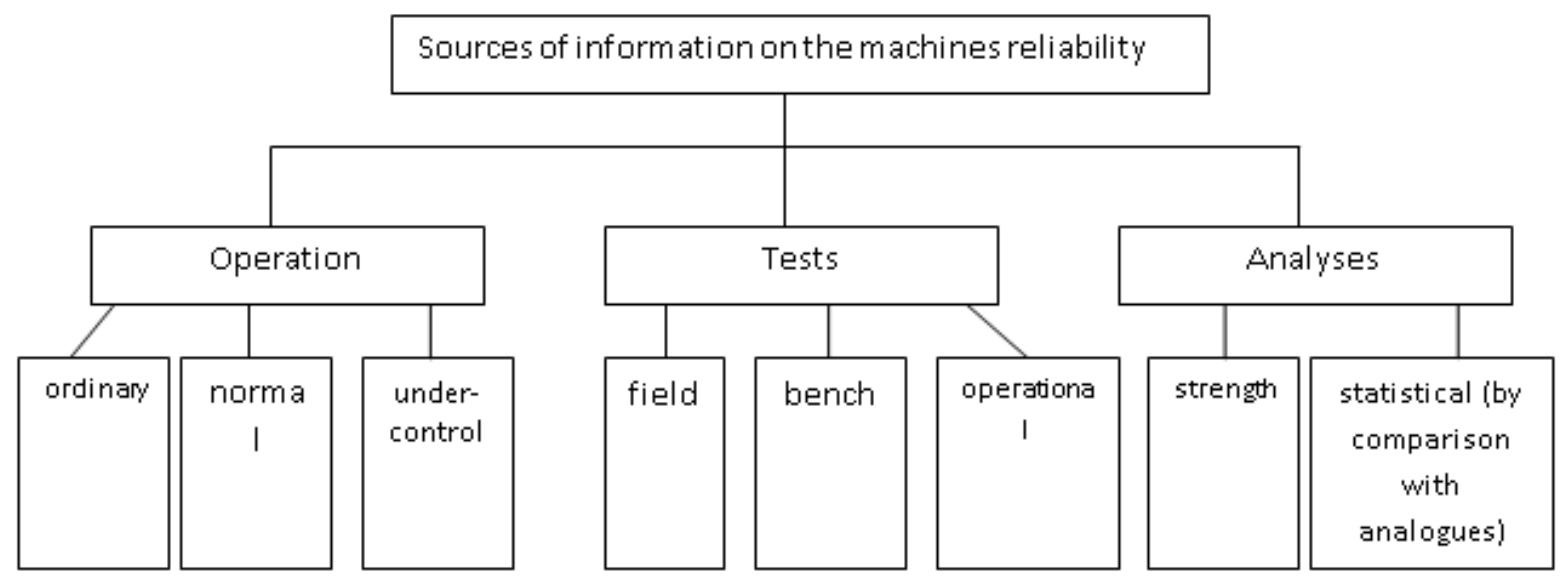

Figure 1. Sources of information on the machines reliability

Random variable is a variable that, as a result of tests, may take one of the possible values unknown beforehand. In reliability theory, such random variables as resource, time to failure, number of failures during a certain period of operation, recovery time, etc. are used. Random variable is said to be continuous if it can take any value within one or more specified intervals as a result of tests. Random variable is called discrete if it can take a finite or infinite countable set of values, that is, if these values can be numbered in some order. Discrete random variables include data of statistical (empirical) observations of object failures, i.e. time to failure, number of failures, etc.

To fully characterize a random variable, it is necessary to specify not only its all possible values, but also the law of its distribution.

Distribution law is a dependence that establishes a relationship between the possible values of a random variable and their probabilities. When calculating reliability, determining the distribution law is a required procedure for obtaining initial data in order to calculate reliability indices. 
The law of continuous random variable distribution is predetermined by the distribution function $\mathrm{F}(\mathrm{x})$, which is also called a cumulative distribution function. It determines the probability that the random variable $X$ will take a value less than $x$ as a result of the test, that is

$$
F(x)=P(X<x) \text {. }
$$

The $F(x)$ function is a non-decreasing $x$ function (monotonically increasing for continuous processes and stepwise increasing for discrete processes). It varies from 0 to 1 within the variation limits of the random variable $X$.

The reliability theory uses the term "time" (time to failure, serviceable life), that is some random value $\tau$, and failure is represented as an event consisting in the fact that $\tau<t$. In this case, the $F(x)$ function of the running time $\tau$ has a physical meaning of the object failure probability in a time less than $t$ :

$$
F(t)=P(\tau<t) \text {. }
$$

The derivative of distribution function per current variable (time $t$ ) is called distribution density:

$$
f(t)=d F(t) / d t
$$

It characterizes the repetition frequency of the given random variable value. In some cases, it is sufficient to characterize the distribution of a random variable via some numerical values, mathematical expectation (mean value), mode and median characterizing the position of the centers of random variables grouping along the axis of abscissas, dispersion, mean square deviation, and a variation coefficient characterizing the dispersion of the random variable (Pronikov, 2002).

The mathematical expectation (mean value) $M_{t}$ is the major and simplest characteristic of random variable $t$. The mathematical expectation value determined based on observation results both for discrete and continuous quantities is called an evaluation of mathematical expectation or an evaluation of mean value $T$ :

$$
T=\sum \frac{t_{1}}{N} \text { or } T=\sum \frac{t_{1} \cdot g_{1}}{N}
$$

where $N$ is the total number of observations; $t_{l}$ is the value of the random variable; $g_{l}$ is the number of identical values $t_{l}$.

It is assumed that $M_{t}=T$ at a sufficiently large number of tests (observations).

The mathematical expectation is determined depending on the distribution density $f(t)$ (for continuous values) or the probability $p_{l}$ of value $t_{l}$ occurrence (for discrete quantities) in probabilistic problems:

$$
M_{t}=\int_{-\infty}^{+\infty} t f(t) d t \text { or } M_{t}=\sum t_{1} \cdot p_{1}
$$

The variance of random variable is a mathematical expectation of this value deviation square from its mathematical expectation. The variance estimate of a random variable is the mean value of the square of difference between the values of random variable and its mean value (Amalitsky et al, 2002):

$$
D_{t}=\frac{1}{N-1} \cdot \sum\left(t_{1}-T\right)^{2} \text { or } D_{t}=\frac{1}{N-1} \cdot \sum g_{1} \cdot\left(t_{1}-T\right)^{2}
$$

For continuous random variables: 


$$
D_{t}=\int_{-\infty}^{+\infty}\left(t-M_{t}\right)^{2} f(t) d t
$$

For discrete random variables:

$$
D_{t}=\sum\left(t-M_{t}\right)^{2} \cdot p_{i}
$$

Since it is more convenient to use the dispersion characteristic of a random variable having the same dimension as a random variable, the following characteristic as a mean square deviation shall be introduced:

$$
S_{t}=\sqrt{D_{t}}
$$

To estimate the dispersion using a dimensionless (relative) value, the coefficient of variation equal to the ratio of the mean square deviation to the mathematical expectation is applied:

$$
v_{t}=\frac{S_{t}}{M_{t}}
$$

Quantile is the value of a random variable corresponding to a specified probability.

Median is a quantile corresponding to a probability of 0.5 . The median characterizes the location of the center of a random variable grouping. The area under the density distribution function graph is divided in half by the median.

Mode of a random variable is its most probable value, or, in other words, the value at which the probability density is maximum.

Reliability parameters are used for state assessment in statistical interpretation and for forecasting in probability interpretation. The first ones are expressed in digital numbers; they are called as estimates in the probability theory and mathematical theory of reliability. They are taken as true reliability characteristics at a sufficiently large number of tests.

Determining the serviceable life of an element or time to failure of an object, as well as other random variables, such as recovery time, accuracy resource, overhaul life, etc. based on statistical data of probability distribution law is one of the required operations when analyzing and calculating reliability. Distribution laws, such as exponential, normal, and Weibull distribution law (Reshetov, 1988), are mainly used when calculating the reliability of machinery and equipment used in the forest sector.

Exponential law is characterized by the density of the time-to-failure distribution (the density of failure probability)

$$
f(t)=\lambda \cdot \exp (-\lambda t)
$$

where $\lambda$ is the failure rate (distribution law parameter). The probability of failure-free operation (reliability function) (Fig. 2, b) (Amalitsky et al, 2002):

$$
P_{t}=\int_{l}^{+\infty} f(x) d x=\exp (-\lambda t) .
$$


The probability of failure occurrence shall be determined by the following formula:

$$
F(t)=\int_{0}^{t} f(x) d(x)=1-p(x)
$$

Mean time of a product to failure:

$$
T=\int_{0}^{\infty} P(t) d(x)=\frac{1}{x} .
$$

Dispersion of a product time to failure:

$$
D_{t}=\int_{0}^{\infty}(t-T)^{2} f(t) d t=\frac{1}{\lambda^{2}}
$$

Mean-square deviation of the time to failure:

$$
S_{t}=\sqrt{D_{t}}=\frac{1}{\lambda}
$$

In terms of exponential law, failure rate is a constant value inversely proportional to the time to failure or the mean time of failure-free operation:

$$
\lambda(t)=\lambda=\frac{1}{T}=\text { const } .
$$

This means that the previous use of an object up to some moment in time $t$ does not affect the residual time of nonfailure operation. Hence, while in operation, the object is not influenced by the wear-out effect, that is, it does not age. Therefore, this law is not applicable for the failures associated with the wear of rubbing surfaces. The law is typical for sudden, emergency failures (for example, during running-in period) associated with breakages and destructions of an object. It is especially important that the time-tofailure distributions of complicated recoverable systems consisting of many elements (Amalitsky et al, 2002) correlate with the exponential law very accurately. Since at $t=T$, the probability $P(T)=0.37,63 \%$ of failures occur for the time $t \leq T$, and only $37 \%$ occur later. Thus, to ensure the required probability of failure-free operation of 0.9 or 0.99 , a small amount of the mean time to failure $(0.1$ and 0.01 , respectively) may be used only.

If the operation of an object occurs under different modes, and consequently, under different failure rates $\lambda_{1}$ (for the time $t_{1}$ ) and $\lambda_{2}$ (for the time $t_{2}$ ), then the following may be obtained from the theorem of multiplication of probabilities:

$$
P(t)=\exp \left[-\lambda_{1} \cdot t_{1}+\lambda_{2} \cdot t_{2}\right]
$$

With high requirements to object failure-free performance, the period of its continuous operation, that is, its resource $T_{\mathrm{p}}$ is limited to a certain value of the permissible probability of failure-free operation $P\left(T_{p}\right)$. 
Application of the exponential law for the objects with high failure-free performance (Pronikov, 2002) can be considered as acceptable and justified.

The normal law describes well the distribution of the probabilities of the time to failure, the resource of elements and other reliability indices when they depend on a large number of random factors being uniform in their influence, the effect of each of which is insignificant compared to the totality of all the others. This law is typical for gradual failures caused by wear and aging. Normal distribution law determines the time to failure of many repairable and non-repairable objects, the dimensions and errors in the measurements of parts. Normal distribution is the most universal law and is widely used for practical calculations.

The density of normal distribution (Fig. 3, a) (Amalitsky et al, 2002]:

$$
f(t)=\frac{1}{S \cdot \sqrt{2 \pi}} \cdot \exp \left(-\frac{(t-T)^{2}}{2 S^{2}}\right)
$$

where $T$ is the mathematical expectation of the time to failure; $S$ is the mean square deviation of the time to failure.

In this case, it is necessary to consider that the area under the curve $f(t)$ is equal to

$$
\int_{-\infty}^{+\infty} f(t) d(x)=1
$$

Thus, it may be concluded that all $100 \%$ of the elements will have time to failure or a serviceable life, ranging from $-\infty$ to $+\infty$. If we integrate the density function within the range from $(T-3 S)$ to $(T+3 S)$, we will get the value of 0.9973 . This is the characteristic feature of the normal distribution law: virtually, all the dispersion $(99.73 \%$ ) of a random variable lies within the interval of $T \pm 3 S$, that is, around the center of grouping within the limits of six mean quadratic deviations. As a result, knowing $T$ and $S$ values, one can practically determine the range of possible values of the random variable.

The probability of failure-free operation (reliability function) shall be determined by the following formula:

$$
P(t)=\frac{1}{S \cdot \sqrt{2 \pi}} \int_{t}^{\infty} \exp \left(-\frac{(x-t)^{2}}{2 S^{2}}\right) d x
$$

The probability of failure occurrence shall be determined by the following formula:

$$
F(t)=\frac{1}{S \cdot \sqrt{2 \pi}} \int_{0}^{\infty} \exp \left(-\frac{(x-t)^{2}}{2 S^{2}}\right) d x=1-P(t)
$$

The integration limits of the last two expressions show that at an arbitrarily taken time point $t$, the part of the area under the density function curve within the limits from 0 to $t$ will characterize the relative number of out-of-operation (failed) components, and the part of the area within the limits from $t$ to $\infty$ will characterize the relative quantity of the components remaining serviceable by the time $t$, that is, the probability of failure-free operation.

In order to simplify the calculations, centered and standardized normal distribution shall be used, in which the grouping center coincides with the origin of coordinates, and the abscissa values are expressed in the 
fractions of $S$. The normalization consists in the change from $t$ value to the auxiliary linear function $z=(t-$ $T) / S$.

The density of normalized distribution:

$$
\varphi(z)=\frac{1}{\sqrt{2 \pi}} \cdot \exp \left(-\frac{z^{2}}{2}\right),-\infty<z<+\infty
$$

The normalized distribution function:

$$
\Phi(z)=\frac{1}{\sqrt{2 \pi}} \cdot \int_{0}^{z} \exp \left(-\frac{z^{2}}{2}\right) d z
$$

This function is called a normalized Laplace function. We have $\Phi(-Z)=-\Phi(Z)$ at negative values of $Z$. To calculate the Laplace function, Table 1 was complied. The probability of failure-free operation $P(t)$ is expressed in terms of the Laplace function as follows

$$
P(t)=0.5+\Phi \cdot\left(\frac{t-T}{S}\right)=0.5+\Phi(z) .
$$

Since the duration of the object trouble-free operation cannot be negative, the normal distribution in general terms can be applied to reliability problems in case of $S<<T$ only. If the condition is not fulfilled, then the truncated normal law is applied. The essence of the truncation lies in the fact that all values $t<0$ and $f(t)=0$ at $\mathrm{t}<0$ (Gnedenko, 1966) are excluded from the set of the random variable values.

Table 1. Values of the normalized Laplace function (Engineering methods for ensuring system reliability) $\Phi(z)$

\begin{tabular}{cccccccc}
\hline$Z$ & $\Phi(z)$ & $z$ & $\Phi(z)$ & $z$ & $\Phi(z)$ & $z$ & $\Phi(z)$ \\
\hline 0.0 & 0.000 & 1.0 & 0.341 & 2.0 & 0.477 & 3.0 & 0.49865 \\
0.1 & 0.040 & 1.1 & 0.364 & 2.1 & 0.482 & 3.1 & 0.49903 \\
0.2 & 0.079 & 1.2 & 0.385 & 2.2 & 0.486 & 3.2 & 0.49931 \\
0.3 & 0.118 & 1.3 & 0.403 & 2.3 & 0.489 & 3.3 & 0.49952 \\
0.4 & 0.155 & 1.4 & 0.419 & 2.4 & 0.493 & 3.4 & 0.49966 \\
0.5 & 0.191 & 1.5 & 0.433 & 2.5 & 0.493 & 3.5 & 0.49977 \\
0.6 & 0.226 & 1.6 & 0.445 & 2.6 & 0.495 & 3.6 & 0.49984 \\
0.7 & 0.258 & 1.7 & 0.455 & 2.7 & 0.4965 & 3.7 & 0.49989 \\
0.8 & 0.288 & 1.8 & 0.464 & 2.8 & 0.4974 & 3.8 & 0.49993 \\
0.9 & 0.316 & 1.9 & 0.471 & 2.9 & 0.4981 & 3.9 & 0.49995 \\
\hline \multicolumn{2}{r}{ Note: $\Phi(-\mathrm{z})=-\Phi(\mathrm{z})$} & & & & & &
\end{tabular}

The density function of the truncated normal distribution law is as follows:

$$
f(t)=\frac{A}{S \cdot \sqrt{2 \pi}} \cdot \exp \left(-\frac{(t-T)^{2}}{2 S^{2}}\right) .
$$


where $A$ is a normalizing constant factor.

The values of $A$ shall be calculated using the following formula:

$$
A=\frac{1}{0.5+\Phi \cdot\left(\frac{T}{S}\right)} .
$$

where $\Phi(T / S)$ is the Laplace function.

The truncated normal distribution describes the reliability analysis results of the complicated systems with a good accuracy, taking into account the drift of the elements parameters (accuracy, vibration strength, temperature, etc.) beyond the allowable limits during operation. Nevertheless, at $T / S>2$, which is the case in the majority of cases when calculating the reliability of machines under consideration with the normal time-to-failure distribution, factor $A$ is differs little from unity, and the truncated normal distribution is described by the ordinary normal law quite accurately.

\section{RESULTS AND DISCUSSION}

The Weibull distribution is rather universal covering a wide range of probability change cases via varying parameters. It describes well the fatigue failures resulting from the combined action of wear and impact loads, for example, the failures of rolling bearings, objects consisting of duplicated components connected in series. It is applied for reliability assessment of machines parts and components, in particular, vehicles, carrying and lifting machines as well as others. It is also used for reliability assessment regarding failures that arise during the process of aging of the product (Amalitsky et al, 2002).

The probability density of time to failure for the Weibull distribution is as follows:

$$
f(t)=\frac{b}{a} \cdot(t-a)^{b-1} \exp \left(-\left(\frac{t}{a}\right)^{b}\right)
$$

where $a$ is the scale parameter (sets the scale of the distribution curve along the abscissa axis); $b$ is the form parameter (determines the sharpness and asymmetry of the distribution density curve).

Probability of product trouble-free operation:

$$
P(t)=\exp \left(-\left(\frac{t}{a}\right)^{b}\right)
$$

Probability of failure occurrence:

$$
F(t)=1-\exp \left(-\left(\frac{t}{a}\right)^{b}\right)=1-P(t)
$$

Rate of failures:

$$
\lambda(t)=\frac{f(t)}{P(t)}=\frac{b}{a} \cdot\left(\frac{t}{a}\right)^{b-1} .
$$


The probability density and the failure-free operation probability curves are shown in Fig. 3 (a, b).

The quantities $a$ and $b$ are always positive ones. The Weibull distribution becomes exponential at $b=1$, which is thus a special case of Weibull distribution. The failure rate $\lambda(t)$ becomes a decreasing function of time at $b<1$, so the Weibull law with the parameter $b<1$ can be used for estimating the reliability of objects during their running-in. At $b>1$, the Weibull distribution is characterized by the failure rate increasing function, and it is more convenient to use it for estimating the reliability of failures caused by wear. The Weibull distribution is close to normal distribution and the probability density function takes on a bell form at $b=3.5 \ldots 4.0$.

The Weibull distribution is widely used in reliability calculations, since it is possible to achieve a more complete correspondence of the theoretical distribution law to the experimental data (Reliability of forest sector machinery and equipment, 2002) via selecting the $a$ and $b$ parameters.

Taking the logarithm of the expression for calculating the probability of failure-free operation $P(t)$, the following can be obtained:

$$
\ln \{-\ln (1-F(t))\}=b \cdot \ln t-b \cdot \ln a .
$$

This equation characterizes the linear dependence of its left part on $\ln t$. Having denoted $y=\ln \{-\ln [-$ $F(t)]\}, A=-b \cdot \ln a, B=b, \mathrm{x}=\ln t$, we can write the straight line equation as $y=A+B x$.

The principle of the least square method is that the coefficients $A$ and $B$ that define the straight-line equation describing the location of the experimental points $x_{i}$ and $y_{i}$ in the graph in the $x$ and $y$ coordinate system in the best possible way are determined per the totality of values $x_{i}$ and $y_{i}$ calculated from the sample data. In this case, the sum of squared deviations of the experimental points from the straight line $y$ $=A+B x$ turns to a minimum (Reshetov, 1999).

Let the variational series of times to failure $t_{1}, t_{2}, \ldots t_{i}, \ldots t_{n}$ be obtained in the process of testing the objects for reliability (in the order of increasing the numerical values).

The values of $x_{i}$ and $y_{i}$ shall be calculated based on the data of variational series. In this case

$$
\begin{gathered}
x_{i}=\ln t_{i} ; \\
y_{i}=\ln \left\{-\ln \left[1-\hat{F}\left(t_{i}\right)\right]\right\}, l=1,2 \ldots n,
\end{gathered}
$$

where $t_{i}$ is the $i$-th value of the time to failure variation series, $\hat{F}\left(t_{i}\right)$ is the distribution function statistical value corresponding to $t_{i}$, and $n$ is the number of sample values.

The values $\hat{F}\left(t_{i}\right)$ shall be calculated according to the following formula:

$$
\hat{F}\left(t_{i}\right)=\frac{(t-0.5)}{n} .
$$

where $i$ is the consecutive number of the variational series of times to failure located in ascending order.

To find the coefficients $A$ and $B$, we shall compile a table in which the values of $t_{i}$ of variational series are written, and then $\hat{F}\left(t_{i}\right), x_{i},\left(x_{i}-\bar{x}\right),\left(x_{i}-\bar{x}\right)^{2}, y_{i},\left(y_{i}-\bar{y}\right)$ and $\left(x_{i}-\bar{x}\right) \cdot\left(y_{i}-\bar{y}\right)$ shall be calculated for each of these values, as well as added to Table 1.

The values $\bar{x}$ and $\bar{y}$ shall be determined from the following expressions: 


$$
\bar{x}=\frac{1}{n} \sum_{i=1}^{n} x_{i} ; \quad \bar{y}=\frac{1}{n} \sum_{i=1}^{n} y_{i}
$$

The coefficient $B$ shall be calculated from the following formula:

$$
B=\frac{\sum_{i=1}^{n}\left(x_{i}-\bar{x}\right) \cdot\left(y_{i}-\bar{y}\right)}{\sum_{i=1}^{n}\left(x_{i}-\bar{x}\right)^{2}}
$$

Then the coefficient $A$ shall be calculated from the following expression:

$$
A=\bar{y}-B \bar{x}
$$

The parameters of the Weibull law shall be found based on the values of $B$ and $A$ :

$$
b=B, a=\exp \left(-\frac{A}{B}\right)
$$

Sudden failures are formed as a consequence of the effect of overloads, which are associated with the inevitable random fluctuations of environmental conditions and inevitable fluctuations in component interaction.

If there is a load fluctuation $R(t)$ near the mean level $R$ in a random stationary process, then a moment $t_{0}$, when it reaches a value exceeding the permissible limit level $R_{n}$, is possible and a failure occurs then. This process is called an instantaneous damage scheme.

The flow of sudden failures, as a rule, is described with a sufficient accuracy by the exponential distribution law with constant intensity $(\lambda=$ const), and the probability of failure-free operation shall be described by the following equation:

$$
P(t)=e^{-\lambda t}
$$

where $\lambda=1 / T$ is a failure flow parameter; $T$ is a mean lifetime (time to failure) of the element; $t$ is the time for which $P(t)$ has to be determined.

The formula (38) can be represented in the following form for the values of $P(t)>0.9$ with the accuracy sufficient for practice:

$$
P(t)=1-\lambda \cdot t=1-\frac{t}{T}
$$

When using the exponential distribution law, it is necessary to point out that the condition $\lambda=$ const remains for a certain period of time.

Gradual failures of machine elements occur as a result of accumulative damages. The change in the initial quality of an element is most frequently caused by wear or accumulation of fatigue damages. As a result of these processes, a part loses its original strength, and its breakdown or plastic deformation (functional failure) 
takes place. It is possible that the change in the initial quality of an element will lead to an increase in the processing error or will be the cause of an increased vibration, etc. (parametric failure) (Amalitsky et al, 2002). Professor A. S. Pronikov (2002) has developed a gradual failure formation model for the most common case when the change in the parameter $x$ of an object is proportional to the rate $\gamma$ of the change process and the operating time $t(x=\gamma t)$.

Accumulation of damages is characterized by a monotonous random variation of the parameter $x(t)$ in time due to both external factors and the progress of physical processes occurring inside the element. Therefore, the most typical one is the case when normal distribution law determines velocity with the following density:

$$
f(\gamma)=\frac{1}{S_{\gamma} \sqrt{2 \pi}} \cdot \exp \left[-\frac{\left(\gamma_{x}-\bar{\gamma}\right)^{2}}{2 S_{\gamma}^{2}}\right]
$$

where $\bar{\gamma}$ is the average value (mathematical expectation) of the damaging process speed or change in the output parameter; $S_{\gamma}$ is the mean root square deviation.

The maximum permissible value of the parameter $x_{\max }$ determines the life time (time to failure) of the element $t=T$, which is a function of a random argument $\gamma$,

$$
T=\varphi(\gamma)=\frac{x_{\max }}{\gamma}
$$

The mean lifetime shall be determined by the following formula:

$$
\bar{T}=\frac{x_{\max }}{\gamma}
$$

The problem consists in finding the distribution density $f(t)$ based on the given function $f(\gamma)$ (Fig. 5, a). The following formula shall be applied for the functions of the random argument in probability theory

$$
f(t=T)=f[\psi(T)]\left|\psi^{\prime}(T)\right|
$$

where $\psi(T)$ is the inverse function of $\varphi(\gamma)$, that is $\psi(T)=\frac{x_{\max }}{T} ; \psi^{\prime}(T)=\frac{x_{\max }}{T^{2}}$ is the derivative of this function.

Substituting these values into the previous expression and making the transformations, we shall obtain the following:

$$
f(T)=\frac{\bar{T}}{\delta \sqrt{2 \pi}} \cdot \frac{1}{T^{2}} \cdot \exp \left[-\frac{(\bar{T}-T)^{2}}{2 \delta^{2} T^{2}}\right]
$$

where $\delta=\frac{S_{\gamma}}{\bar{\gamma}}$ is the variation coefficient. 
We shall introduce the dimensionless time $\tau$ in the fractions of $\bar{T}$ for convenience of calculations

$$
\tau=\frac{T}{\bar{T}}
$$

Then, the design formula will take the following form:

$$
f(\tau)=\frac{1}{\delta \sqrt{2 \pi}} \cdot \frac{1}{\tau^{2}} \exp \left[-\frac{(1-\tau)^{2}}{2 \delta^{2} \tau^{2}}\right]
$$

where $f(\tau)=f(\tau)=\bar{T} f(T)$ and $T=\tau \bar{T}$.

This formula is convenient in that the probability density is a function of one single dimensionless parameter, i.e. the variation coefficient $\delta$.

To determine failure probability $F(T)$, it is necessary to integrate the probability density function

$$
F(T)=\int_{0}^{\tau} f(t) d t=\int_{0}^{\tau} f(x) d x=F(\tau)
$$

If we introduce the variable $z=(1-\tau) / \delta \tau$, this integral will be reduced to the Laplace function. Taking into account the fact that the probability of failure-free operation $P(T)=1-F(T)$, we shall obtain the following:

$$
P(t)=P(\tau)=0.5+\left(\frac{1-\tau}{\delta \tau}\right)
$$

where $P(t)$ is the normalized Laplace function, $0 \leq P(t) \leq 0.5$. $P(t)=1$ at $\tau=0$, and $P(t) \rightarrow 0$ at $\tau \rightarrow \infty$ Having expressed $P(T)$ via the parameters $\lambda_{\max }, \bar{y}, S_{\gamma}$, which serve as the initial data while solving the set task, the formula can be written in the other form:

$$
P(t)=0.5+\left(\frac{x_{\max }-\overline{\gamma t}}{t S_{\gamma}}\right)
$$

The analyzed scheme is simplified, since it does not take into account the dispersion of the initial parameters in reality, the type of implementation $x(t)$ depends on the initial state of the element, for example, the quality of manufacture or repair. Considering the dispersion of the initial parameters,

$$
x=x_{0}+\gamma t
$$

where $x_{0}$ is the product initial parameter.

The initial parameter of the element is a random variable and is subjected to a certain distribution law. Serviceable lifetime is a function of two independent random arguments $x_{0}$ and $\gamma$

$$
T=t=\frac{\left(x_{\max }-x_{0}\right)}{\gamma}
$$


If random arguments $x_{0}$ and $\gamma$ are distributed according to the normal law, then the parameter $x$ for each value $t=T$ will be distributed according to the same law with the same parameters (Amalitsky et al., 2002).

Mathematical expectation

$$
x=x_{0}+\gamma T
$$

Mean square deviation

$$
S_{x}=\sqrt{S_{0}^{2}+\left(S_{\gamma} T\right)^{2}}
$$

where $x_{0}$ is the mathematical expectation; $S_{0}$ is the root-mean-square deviation of the random parameter $x_{0}$. The probability of failure-free operation:

$$
P(t)=0.5+\Phi\left[\frac{x_{\max }-x_{0}-\overline{\gamma t}}{\sqrt{S_{0}^{2}+S_{\gamma}^{2} \cdot t^{2}}}\right]
$$

The same formula can also be used in the nonlinear progress of parameter change process, that is, when there is mathematical expectation $\bar{\gamma}(t)$ and the dispersion $S_{\gamma}^{2}(t)$ is the function of time in some cases. Thus, the change in the output parameter can be written for any regularity in the general form.

$$
P(t)=0.5+\Phi\left[\frac{x_{\max }-x_{0}-\bar{\gamma}(t) t}{\sqrt{S_{0}^{2}+S_{\gamma}^{2}(t) \cdot t^{2}}}\right]
$$

The formula (54) makes it possible to solve the so-called "direct" problem, i.e. to determine the probability of failure-free operation $P(t)$ at a specified life.

To solve the "inverse" problem, i.e. the determination of the serviceable lifetime $T$ at the given level $P(t)$, the value $t=T$, which is included into the argument of the Laplace function, is the desired quantity in the formula (54). This argument will serve as a quantile $u_{a}$ of the normal distribution, that is, it will be the value that corresponds to the given probability $P(t)$.

Having equated the value of the argument of function $\Phi$ to $x_{P}$ from the formula (54), we shall obtain the quadratic equation for determination of $T$ :

$$
x_{p} \sqrt{S_{0}^{2}+S_{\gamma}^{2} \cdot t^{2}}=x_{\max }-x_{0}-\bar{\gamma} t
$$

The calculation procedure lies in the fact that we shall find the corresponding value $x_{p}$ for the given value of $P(t)$ based on the tables for quantiles of the normal distribution, and we shall find the serviceable lifetime $t=T$ from the equation (56).

The failure occurrence scheme as a result of effect of several causes, for example, the load $R(t)$ of a stationary random process with the maximum permissible load level $R_{n}(t)$ decreasing due to wear is the most typical for machine real elements. Determining the physical nature of such failures is difficult, so they are mistakenly referred to usual gradual or (which is more often) to sudden failures. 
The strength decreases with time at any system subject to wear, aging, corrosion, etc. The system retains the original properties at $t \ll t_{0}$. The maximum permissible load $R_{n}(0)$ is large, and the probability of failure is small. $R_{n}$ decreases to a level of $R_{l n}$, which is more likely to be lower than the workload at a time close to $t_{0}$ and especially after it.

The service life of the elements, whose failures arise as a result of the implementation of several causes action scheme, can often be described via the Weibull distribution with an adequate degree of accuracy.

The experimental determination of reliability is based on the registration of times to failure occurrence. Such events are accidental, since inevitable changes in production factors and operating conditions lead to dispersion of indices characterizing the technical condition of a vehicle. In this regard, such indices reach the limit values at different values of operating time (running), that is why the group reliability factor, i.e. the time to failure can be considered as a set of probabilistic static characteristics obtained while processing the databank samples based on the probability theory and mathematical statistics.

The parameters of the time to failure distribution law are the initial data for determining the mean time to failure of controllable products. The calculation of the time to failure consists in constructing variational series to be determined by the following indices: $l_{\max }$ is the maximum time to failure or suspended time to failure; $\Delta l$ is the value of the variational series interval; $K$ is the number of intervals; $n_{i}$ is the number of products with this failure included in the $i$-th interval; $D$ is the number of products with suspended time to failure included in the $i$-th interval.

The cumulative frequency $m_{n i}$ shall be determined for each interval.

If there are no suspended lifelengths within the interval preceding the $i$-th one, then the cumulative frequency shall be determined by the following formula:

$$
m_{n i}=\sum_{1}^{i} n_{i}
$$

If there are suspended lifelengths within the interval preceding the $i$-th one, then the cumulative frequency shall be determined by the following formula:

$$
m_{n i}=m_{n(i-1)}+\Delta i_{n i}
$$

where $m_{n(i-1)}$ is the cumulative frequency referred to the upper boundary of the interval preceding the one under consideration; $\Delta i$ is the failure weight within the interval,

$$
\Delta i=\frac{N+1-m_{n(i-1)}}{N+1-\sum_{1}^{i-1}(n+1)}
$$

where $N$ is the sample size.

The time to failure of a single element of the mechanical system structural scheme of a product shall be determined by the following formula:

$$
L_{0}=\frac{l}{T}
$$

where $l$ is the time of a restorable product to the first failure; $T$ is the mathematical expectation of the number of failures. 
Time to failure depends on the running interval at which it is being determined. It is reasonable to define the vehicles reliability factors via the time to failure $L_{o}$ not depending on the random value of the time to the first failure, but on the fixed interval $\Delta l$. In this regard, the problem of finding the value of time to failure is reduced to determining the value of the mathematical expectation of the number of failures $T$, which can be determined from the following expression:

$$
T=F_{i}(l)+\int_{0}^{1} H(l-x) \cdot d F_{i}(x)
$$

or according to the following formula:

$$
H(l)=\sum_{i=1}^{\infty} F_{i}(l)
$$

where $F_{i}(l)$ is the distribution function of the time to the $i$-th failure to be determined from the relation:

$$
F_{i}(l)=\int_{0}^{1} F_{i-1}(l-x) d F(x)
$$

wherein $F(l)=F_{i}(l)$ is the distribution function up to the first failure, while $l$ varies from 2 to $\infty$. $f(x)$ is the differential distribution function,

$$
d F(x)=f(x) d x
$$

The general formula for the approximate definition of $F_{l}(t)$ for any 1 can be represented in the following form (Trikozyuk, 1980):

$$
\begin{gathered}
F_{i}(t)=\Delta x\left[0.5 \cdot F_{i-1}\left(l-x_{0}\right) \cdot f\left(x_{0}\right)+F_{i-1}\left(l-x_{n}\right) \cdot f\left(x_{n}\right)+F_{i-1}\left(l-x_{1}\right) \cdot f\left(x_{1}\right)+\right. \\
\left.+F_{i-2}\left(l-x_{2}\right) \cdot f\left(x_{2}\right)+\ldots+F_{i-2}\left(l-x_{n-2}\right) \cdot f\left(x_{n-2}\right)\right]
\end{gathered}
$$

where $n$ is the number of intervals into which the entire integration range from $\mathrm{x}_{0}$ to $\mathrm{x}_{\mathrm{n}}$ is divided; $d x$ is the fixed value of a single interval assumed equal to $5000 \mathrm{~km}$ in these calculations; $x_{0}$ and $x_{n}$ are the initial and final points of the integration range, respectively.

$$
x_{0}=0 ; \quad x_{n}=\Delta x_{n} ; n=\frac{t}{\Delta x} .
$$

As it was established (Cline, 1968), the failure flow of the elements operating under linear deformation conditions shall be distributed according to the Weibull law.

Consequently, the integral redistribution function has the following form: 


$$
F(x)=1-\exp \left[-\left(\frac{x}{a}\right)^{b}\right]
$$

where $a$ and $b$ are the Weibull distribution parameters.

The differential function of this distribution has the following form:

$$
f(x)=\frac{b}{a} \cdot\left(\frac{x}{a}\right)^{b-1} \cdot \exp \left[-\left(\frac{x}{a}\right)^{b}\right]
$$

The properties of the Weibull distribution function are as follows:

$$
\begin{gathered}
F_{0}(0)=0 ; \quad f_{0}=0 ; \\
F_{l}(0)=\int_{0}^{l} F_{i-1}(x-l) \cdot f(x) d x=0 ;
\end{gathered}
$$

It is possible to simplify the formulas of determining the distribution function of the time to the lst failure of the object of research for any value of $l=1 ; 2 ; 3$.., which acquires the following form of trapezoid formula (more convenient for numerical integration) after appropriate transformation:

$$
F_{i}(t)=\Delta x \cdot \sum F_{i-1}\left(l-x_{m}\right) \cdot f\left(x_{m}\right)
$$

where $x_{m}=\Delta x_{m} ; n=\frac{l}{\Delta x}$.

In order to determine the value of $F_{i}(l)$ according to the specified trapezoid formula, it is necessary to predetermine the value of the function $F_{i-1}\left(l-x_{m}\right)$ at $\mathrm{n}$ points, that is, at $l-x_{m}=\Delta x, 2 \Delta x, 3 \Delta x \ldots(n-1) \Delta x, n$ $\Delta x$.

The specified formula for determining the value of $F_{l}$ at each point of the integration range separation will have the following form:

$$
F_{i}\left(\Delta x_{k}\right)=\Delta x \cdot \sum_{m=1}^{k-1} F_{i-1}[\Delta x \cdot(k-m)] \cdot f\left(\Delta x_{m}\right) \text {. }
$$

$F_{i}(\Delta x)=0$ is obtained at $k=1$ taking into account (Stal Group OOO). In this regard, the previous formula (Zayats, 2013) is valid at $k=2,3 \ldots$ The value of $F_{i}(x)$ for each particular value of running time $\mathrm{t}$ is calculated until the time when the value of $F_{k}(l)$ becomes less than some small, predetermined positive number, for example 0.001 at a certain $l=k$. The calculated times to failure end in this case. The relative error from the replacement of the sum of infinite series with the sum of a finite number of first terms does not exceed $1 \%$ according to practical calculations of determining the time to failure of automobile semitrailer units. 
According to the results of experimental studies, the time to failure of certain elements, pivots and support plates of semitrailers in particular, is distributed according to the normal law. The times to failure shall be determined by the following formula (Stal Group OOO) for such elements

$$
L_{0}^{i}=l \cdot\left[\sum_{k=1}^{\infty} F_{0}\left(\frac{l-k \cdot a_{i}}{\delta \cdot \sqrt{k}}\right)\right]^{-1}
$$

where $a_{i} \& \delta_{i}$ are the normal distribution parameters of the $i$-th element time to failure; $F_{0}(x)$ is a normal distribution function.

After determining the time to failure of each element of the design diagram of the product according to the formulas (Barash et al.., 2004) and (Kuznetsov, 2001), the time to failure of each assembly unit of the product component parts shall be determined:

$$
L_{0}^{j}=\left(\sum_{j=1}^{N} \frac{n_{j}}{T_{0}^{j}}\right)^{-1}
$$

where $n_{j}$ is the number of the j-th type elements in the given assembly unit; $L_{j}$ is the time to failure of the $\mathrm{j}$-th element of the assembly unit; $N$ is the total number of the elements to be calculated in the given product assembly unit.

Similarly, the time to failure of the entire product is as follows:

$$
L_{0}=\left(\sum_{j=1}^{M} \frac{n_{j}}{T_{0}^{j}}\right)^{-1},
$$

where $n_{j}$ is the number of assemblies of the $j$-th type in the product structural scheme; $T_{j}$ is the time to failure of the $j$-th assembly in the product structural scheme; $M$ is the total number of assemblies to be calculated in the given product.

The results of the present paper were used while determining the reliability of KZAP-9370 and KZAP-9385 semitrailers produced by the Krasnoyarsk automobile trailer plant and operating with KAMAZ-54102 semi-tractors. The semitrailer structural scheme was conveniently divided into a specific number of components, according to which the type of failure was formed. Both the least reliable structural components of the semitrailer and the components with a sufficiently large time to failure were determined as a result of data collection on the life lengths of road trains at the time of the beginning of failures and cases of unserviceable condition with subsequent analytical processing using the presented method. The following results of unit-wise time to failure of KZAP9370 semi-trailer have been obtained on the run of 350 thousand kilometers.

Table 2. Data on the time to failure of KZAP-9370 semi-trailer components on a run of 350 thousand kilometers

\begin{tabular}{rlc}
\hline No. & \multicolumn{1}{c}{ Failure type } & Mean time to failure, $\mathrm{km}$ \\
\hline 1 & Destruction of sides and their fixation elements & 12700 \\
2 & Demolition of frame with a floor plate & 61900 \\
3 & Destruction of a support plate with pivot & 241100 \\
4 & Support device failure & 219100 \\
5 & Destruction of an equalizing beam suspension & 61800 \\
6 & Destruction of axle beams & 345000 \\
7 & Destruction of wheeled braking mechanisms & 82600 \\
8 & Failure of brake pneumatic drive elements & 82500 \\
\hline
\end{tabular}


9 Electrical equipment failure

10 Spare wheel holder failure

Time to failure of a semitrailer as a whole
331300

203200

6450

As Table 2 shows, the time to failure of the semitrailer components is characterized by considerable spread in values, as a result of which, the total time to failure of the whole semitrailer, to be determined by the formula (73), is reduced to $6450 \mathrm{~km}$. In practice, this means that there will be a single failure pertaining to any of the listed ten systems on the specified run with $90 \%$ probability. It is possible to point out to the manufacturer the necessity of further development of a certain type of a mechanical system composite element using the obtained data.

\section{CONCLUSIONS}

As a result of the present work, the theoretical provisions regarding calculating the reliability of vehicles on the unit-wise and assembly-wise levels concerning specific failure type have been obtained. It is possible to perform calculations of the time to failure and between failures according to operational observations over a group of objects under control, in particular, transport vehicles according to the compiled formulas.

RECOMMENDATIONS. The materials of the article can be used when it is necessary to estimate the reliability of a mechanical transport system operating under specific conditions, forming particular conditions for the load modes of parts about which the manufacturer can have rough ideas only. Reliability assessment of components and the machine as a whole can point out to the manufacturer the aspects of work on improving specific parts or components with the view of improving the reliability of the product as a whole.

The article has been written with the support of the agreement on creative cooperation between Voronezh State Forest Engineering University and KAMAZ, PJSC.

\section{REFERENCES}

Amalitsky, V. V., Bondar, V. G., Volobaev, A. M., \& Voyakin, A. S. (2002). Reliability of timber complex machinery and equipment. Moscow: Moscow State Forestry University.

Barash, A. L., Zorin, V. A., Fedorov, V. K., \& Shereshov, P. I. (2044). Fundamentals of machine reliability. Balashikha: VTU.

Barlow, R. E., \& Wu, A. S. (1978). Coherent system with multi-state Components. Mathematical Operation Research, 3(4), 275-281. doi: 10.1287/moor.3.4.275.

Cline, S. J. (1968). Similarity and approximate methods. (I. T. Aladiev, \& K. D. Voskresensky, Trans.). Moscow: Mir.

Dhillon, B. S. \& C. Singh. (1979). On fault trees and other reliability evaluation methods. Microelectronics and Reliability, 19(1-2), 57-63.

Galetto, F. (1977). System Availability and Reliability Analysis SARA. In F. Galetto (Ed.), Proceedings 1977 Annual Reliability and Maintainability Symposium (pp.95-100).

Gmurman, V. E. (2006). Theory of probability and mathematical statistics. Moscow: Higher Education.

Gnedenko, B. V. (1966). Mathematical problems of reliability theory. In B. V. Gnedenko, Yu. K. Belyaev, \& I. N. Kovalenko. Science Results. Probability Theory. Mat. Stat. Series: collection of scientific papers. All-Union Institute of Scientific and Technical Information (pp. 7, 53).

Stal Group OOO: Characteristics of steel 18X2H4BA Access mode: World Wide Web. URL: http://www.gkstal.ru/markastal/element.php?m=18\%D52\%CD4\%C2\%C0. 
Klyuev, V. V. (2003). Reliability of machines. In K. V. Frolov (Ed.), Mechanical Engineering Encyclopedia vol. IV3 (p. 592). Moscow: Mechanical Engineering.

Kuznetsov, E. S. (2001). Vehicle Technical Maintenance. Moscow: Transport.

Maslovsky, E. K. (Ed.). (1984). Engineering methods for ensuring the system reliability. Moscow: Mir.

Matveevsky, V. R. (2002). Reliability of technical systems. Moscow State Institute of Electronics and Mathematics.

Pronikov, A. S. (2002). Parametric reliability of machines. Moscow: Moscow State Technical University named after N.E. Bauman.

Pustylnik, E. I. (1968). Statistical methods of analysis and processing of observations. Moscow: Nauka.

Reshetov, D. N., Ivanov, A. S., \& Fadeev, V. V. (1988). Reliability of Machines. Moscow: Higher School.

Rolling. In The Great Soviet Encyclopedia. Access mode: World Wide Web. URL: http://bse.scilib.com/article093227.html.

Rotenberg, R. V. (1973). Fundamentals of machine reliability. Moscow: Moscow State Automobile \& Road Technical University.

GOST 16468-79 Standards Russian Federation (1979). Data Collection and Processing System. Introduction (p.8.). Moscow: Publishing House of Standards.

R 50-54-82-88 Standards Russian Federation (1988). Reliability in technology. Choice of reservation methods and procedures (Recommendations p. 46). Moscow: Recommendations.

GOST 27.002-89 Standards Russian Federation (1989). Reliability in technology. Basic Concepts. Terms and Definitions. Introduction (pp.1-15). Moscow: Publishing House of Standards.

RD 50-690-89 Standards Russian Federation (1989). Reliability in technology. Methods for estimating reliability indices based on experimental data. (Methodical Guidelines p.23). Moscow: USSR State management committee product quality and standards.

GOST 27.402-95 Standards Russian Federation (1995). Reliability in technology. Test plans for monitoring the mean time to failure (between failures). Part 1. Exponential Distribution. Introduction (p.39). Moscow: Publishing House of Standards.

Stroew, S. N. (1973). Determination of the abrasive wear of non-hardened gears. Maschinenbautechnik, 22(4), 169173.

Trikozyuk, V. A. (1980). Increasing the vehicle reliability. Moscow: Transport.

Volkov, V. S. (1999). Forecasting the reliability of the timber complex transport vehicles. Voronezh: Voronezh State Academy of Forestry Engineering.

Zayats, Yu. A. (2013). Fundamentals of the reliability theory. Ryazan: RVVDKU. 